\title{
Review of: "The composition of human vaginal microbiota transferred at birth affects offspring health in a mouse model"
}

\author{
Laura Dearden ${ }^{1}$, Denise Fernandez-Twinn ${ }^{1}$ \\ 1 University of Cambridge
}

Potential competing interests: The author(s) declared that no potential competing interests exist.

This research paper published in Nature Communications in November 2021 seeks to understand the consequences of artificial perinatal exposure to maternal vaginal microbiota on long-term health outcomes. Delays or disruptions to maternal microbial transmission, as is common among caesarean delivered babies and antibiotic-exposed neonates, is associated with increased risk of sepsis, immune system hypersensitivity, allergy, asthma, altered growth trajectories, and obesity later in life. One option to overcome a lack of bacterial exposure is to manually expose newborn babies to maternal microbiota, however the full consequences of transferring bacterial communities from mother to baby are unknown. For this reason, the practice of "seeding" caesarian delivered babies with maternal vaginal or fecal microbiota, to mimic the bacterial exposure of a vaginal birth, remains controversial. To date studies have reported both positive and negative neonatal outcomes after this procedure (Mueller NT et al, Int. J. Obstet. Gynaecol 2020).

In this paper, the authors have used a protocol that simulates birth associated exposure to human vaginal microbiota by administering vaginal microbiota samples collected from women late in pregnancy to caesarean delivered mice at birth via oral gavage. The researchers have focused on the vaginal microbial communities to which a human newborn is exposed during a vaginal birth that have been shown to persist within the infant intestinal tract during the first few days after birth (Community State Type (CST) I and IV). This was admittedly a reductionist approach in line with their very strict criteria for inclusion/exclusion of vaginal lavage and transplantation. The authors also hypothesized that perturbations to the prenatal environment (for example maternal obesity or a hostile vaginal microbiome) would influence offspring response to postnatal microbial exposure and result in long-term changes to offspring health.

Using their model of exposure to human vaginal microbiota CST I and IV in mice, the authors demonstrate that the growth curve for CST IV-exposed males is steeper than that of CST I-exposed males or caesarean delivered mice administered with a vehicle solution, but not different from vaginally delivered males, suggesting that exposure to CST IV restores postnatal growth rates in caesarean delivered mice. The authors also report that birth associated colonization by human microbial communities is associated with 
shifts in peripheral immune cell composition in neonatal mice (most notably an increase in circulating neutrophils) and CST specific transcriptional changes- particularly in genes related to inflammatory pathways- in the neonatal intestinal tract. The authors hypothesise that these- and other- distinct responses to postnatal exposure to CST I or IV noted throughout the manuscript may stem from the different speed of immune response to distinct strains of bacteria, but this is something that requires further investigation given that in different experimental scenarios, different CST were associated with positive or negative outcomes.

In human and murine newborns, a surge in circulating neutrophils occurs within 72 hours of birth and overlaps with birth-associated exposure to maternal microbiota. To confirm that this surge in neutrophils is dependent on exposure to maternal microbiota, the authors used an established model of perinatal antibiotic exposure whereby pregnant dams are exposed to a cocktail of antibiotics late in gestation to deplete microbiota and prevent vertical transmission. Hemotological and CyTOF analyses demonstrate that circulating neutrophils are reduced in antibiotic exposed males compared to CST I, CST IV and vaginally delivered mice- confirming exposure to maternal microbiota is associated with increased neutrophils shortly after birth, and showing that immune cell populations are influenced by premature birth and mode of delivery.

In utero exposure to adverse maternal environments such as maternal stress, obesity or infection is known to have long-term detrimental effects on offspring health. However, whether prenatal exposures might influence the response to postnatal microbiota exposure is unknown. The authors extended their mouse model to include pre-natal exposure to a maternal high-fat low-fiber diet and sub-optimal bacterial vaginosis, followed by post-natal inoculation with CST IV at birth. This experimental protocol resulted in a striking $60 \%$ mortality rate in male animals. This two- hit model is also associated with broad dysregulation in gene expression in the placenta and neonatal ileum after exposure to the combination of maternal high-fat low fiber diet and bacterial vaginosis, that didn't occur after exposure to one condition alone.

This study is one of the first to effectively model an artificial transfer of human vaginal microbiota to newborns that would otherwise not have received vaginal microbiota due to being born via caesarean, and shows the impact any changes to this normal route of transmission can have on numerous newborn organ systems. Unfortunately, the interesting and novel findings of this paper are tempered by the lack of effective experimental controls. The authors state that as the CST- exposed mice were delivered via caesarean at the pre- term age of embryonic day 18, age-matched vaginally delivered offspring were included in the experiments to control for mode of delivery and premature birth. However, although these mice were the same age post- birth as the CST treated animals when analysed, they were born at term and via a spontaneous vaginal delivery. They do not therefore completely control for any effects on newborn 
development that may be due to the process of premature birth, i.e. a lack of normal labour-induced hormones e.g. fetal cortisol and maternal estrogen in addition to the caesarean and CST exposure.

The observation that CST I and IV treated newborn mice sometimes are phenotypically similar to vaginally delivered control mice, and sometimes with vehicle treated control mice is complicated and suggests there are more factors at play during the transfer of vaginal microbiota from mother to child than these two studied CST. Furthermore, although the authors have reported widespread gene expression changes in a range of tissues including the brain, intestine and placenta, the function of these genes (or gene networks) are not extensively discussed, making it hard to ascertain whether the transcriptional changes in response to microbial exposure are beneficial or not to future health. This information is key in improving our understanding whether inoculation with maternal microbiota is beneficial or not in a clinical setting, and so hopefully something that the authors- or others- will be reporting more on soon. 